\title{
Fractal dimension-based damage imaging for composites
}

\author{
Li Zhou*, Hu Sun and Zhiquan He \\ State Key Laboratory of Mechanics and Control of Mechanical Structures, Nanjing University of Aeronautics and \\ Astronautics, Nanjing, Jiangsu, China
}

Received 6 January 2013

Revised 28 March 2013

Accepted 13 April 2013

\begin{abstract}
In this paper, a damage imaging algorithm based on fractal dimension is developed for quantitative damage detection of composite structures. Box-counting dimension, a typical fractal dimension, is employed to analyze the difference of Lamb wave signals, extract damage feature and define damage index. An enhanced reconstruction algorithm for probabilistic inspection of damage is developed for damage imaging. Experimental investigation in a composite laminate and a stiffened composite panel shows that the developed algorithm could quantitatively predict the location and size of not only single but also multiple damages. The influence of parameters in the developed algorithm on the imaging quality and accuracy is studied, and reference values for parameters are presented.
\end{abstract}

Keywords: Lamb wave, fractal dimension, damage imaging, composite structures, multi-damage detection

\section{Introduction}

Advanced composites have been extensively used in marine and aerospace industries for their attractive characteristics such as high strength/stiffness-to-weight ratios. However, damage tolerance of these structures and use of related properties in cost-effective design are heavily dependent on the fundamental understanding of how various types of damages initiate and grow and how reliably they can be monitored. Conventional non-destructive test (NDT) methods such as through-thickness ultrasonic pulse-echo and scanning are not necessarily the best or the most efficient methods in such effort. Conventional nondestructive methods might require disassembly of individual part which is not practical in integral structures of marine and aerospace vehicles.

Compared with the conventional NDT techniques, the online Lamb wave-based structural health monitoring (SHM) techniques can instantaneously provide reliable and quantitative structural health data for in-service composite structures, and cover a relatively large inspection area [1-3], which have been proved to be an effective and promising technique. One of the common features of such techniques is to develop a real-time imaging method to visualize damage, which provides a quick and direct approach for detecting damage location and severity. Yuan and his coworkers $[4,5]$ performed a prestack reverse-time migration technique to image the delamination in composite laminates. Sohn et al. [6,7], Qiu et al. [8] and Miao et al. [9] applied time reversal process concept to develop baseline and baseline-free techniques to identify damage in composites. Malinowski et al. [10] and Rathod el al. [11] developed new phased array-based methods to estimate the damage in composite panels. However, the analysis and interpretation of Lamb waves can be complicated due to their dispersive and multimodal nature. Due to dispersion

\footnotetext{
* Corresponding author: Li Zhou, State Key Laboratory of Mechanics and Control of Mechanical Structures, Nanjing University of Aeronautics and Astronautics, Nanjing 210016, Jiangsu, China. Tel.: +86 258489 1722; Fax: +86 258489 1422; E-mail: lzhou@ nuaa.edu.cn.
} 
characteristics, the various frequency components of Lamb waves travel at different speeds and attenuate at different rates, causing the shapes of wave packets to change as they propagate through a solid medium. In addition, multiple symmetric and anti-symmetric Lamb wave modes are generated as the driving frequency for wave generation increases. A reconstruction algorithm for probabilistic inspection of damage based on the transducer network [12, 13], by extracting the damage indices and predicting the damage possibility near the transmitter-receiver path, is considered as an interesting and potential technique.

In these damage imaging methods, it is crucial to develop signal processing methods to calculate damage index, for example, wavelet transform [6,8], consecutive outlier analysis [7], cross-correlation coefficient [9], etc. Fractal theory, capturing the worldwide attention with its merit in complicated signals, has been research topics of many scholars in signal processing [14,15]. Li et al. [16], Bai et al. [17] and Hadjileontiadis et al. [18] applied fractal dimension (FD) to calculate damage indices for damage detection of 1D beam structures. However, few studies have been carried out to apply FD for damage detection in aircraft composite structures. The present paper will introduce fractal dimension to analyze the Lamb wave signals, extract the damage index and image the damage in 2D complex composite structures.

The paper is organized as follows: Section 2 deals with how to calculate box-counting dimension, a typical FD, and gives the method to extract damage indices of Lamb waves based on FD. In Section 3, an enhanced reconstruction algorithm for probabilistic inspection of damage is developed for damage imaging. In Section 4, experimental studies for damage detection of two typical composites, i.e., a composite laminate and a stiffened composite panel, are presented to demonstrate the feasibility and effectiveness of FD-based imaging algorithm. Finally, some conclusions are drawn in Section 5.

\section{Damage index based on fractal dimension}

\subsection{Fractal dimension}

In mathematics and physics, the dimension of a space or object is defined as the minimum number of coordinates needed to specify each point within it. In Euclidean geometry, a line has a dimension of one because only one coordinate is needed to specify a point on it. A surface has a dimension of two because two coordinates are needed to specify a point on it. However, Euclidean geometry lacks the ability to describe the irregular or fragmented shape of natural features as well as other complex objects. The mathematician Mandelbrot [19] used the adjective "fractal" to indicate objects whose complex geometry cannot be characterized by an integral dimension. Mandelbrot elaborated that when divided into parts, each part of geometric shapes would be a reduced-size copy of the whole [20]. In fractal geometry, the fractal dimension can be considered as an indication of how completely a fractal appears to fill space. For a line, fractal dimension simply gives the number of ordinary dimensions as one because there is only one way to move on a line. However, curves which are self-similar fractal have non-integer fractal dimensions, because they can be considered as ranging from being a one-dimensional line to being nearly plane filling, which is to say that the curves turn and twist so much that they have the potential to visit nearly each part of certain regions of the plane, becoming almost two-dimensional. Correspondingly fractal dimension illustrates the values ranging up from just above 1 to just below 2. Methods for calculating fractal dimensions include Hausdoff dimension, similar dimension, box-counting dimension, etc.

In the present paper, the box-counting dimension is used to calculate the dimensions of Lamb waves. The boxcounting dimension of a set $A$ contained in the space $\boldsymbol{R}^{n}$ is defined as follows: For any $r>0$, let $N_{r}(A)$ be the minimum number of $n$-dimensional cubes of side-length $r$ needed to cover $A$. If there is a number of $d$ so that

$$
N_{r}(A) \propto 1 / r^{d} \text { as } r \rightarrow 0,
$$

where $d$ is the box-counting dimension of $A$.

Note that the box-counting dimension is $d$ if and only if there is some positive constant $k$ so that

$$
\lim _{r \rightarrow 0} \frac{N_{r}(A)}{1 / r^{d}}=k
$$




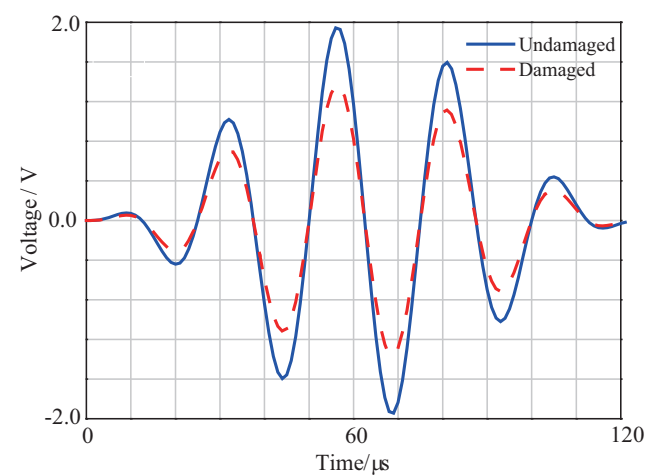

(a)

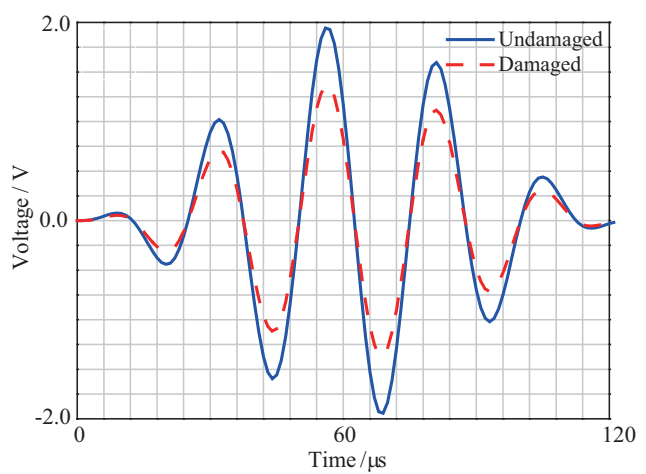

(b)

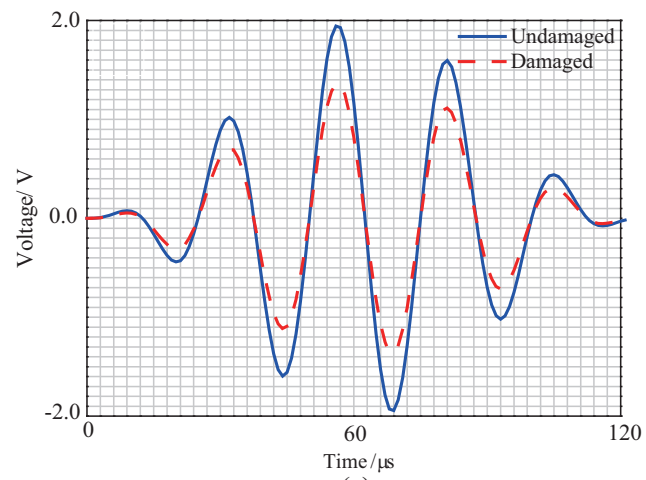

(c)

Fig. 1. Calculating box numbers at different box size for signals: (a) $r=r_{1}$; (b) $r=r_{2}$; (c) $r=r_{3}$. $\left(r_{1}>r_{2}>r_{3}\right.$ ).

Since both sides of Eq. (2) are positive, it will still hold if taking the logarithm of both sides to obtain

$$
\lim _{r \rightarrow 0}\left(\log N_{r}(A)+d \log r\right)=\log k
$$

Solving for $d$ gives

$$
d=\lim _{r \rightarrow 0} \frac{\log k-\log N_{r}(A)}{\log r}=-\lim _{r \rightarrow 0} \frac{\log N_{r}(A)}{\log r}
$$

Note that the term $\log k$ drops out, because it is constant while the denominator becomes infinite as $r \rightarrow 0$. Also, since $0<r<1, \log r$ is negative, $d$ is positive.

In practice, the box-counting algorithm estimates fractal dimension of the curve by counting the number of boxes required to cover the curve for several box sizes, and fitting a straight line to the log-log plot of $N_{r}(A)$ versus $r$. The slope of the least square best fit straight line is taken as an estimate of the box-counting dimension $d$ of the curve.

\subsection{Damage index}

In the active Lamb wave monitoring technique based on the transducer array, piezoelectric ceramic is often used to transmit/receive Lamb wave. If damage is present, compared to undamaged status, Lamb wave signal is scattered and converted into other wave modes. These changes can be identified by calculating FD. Calculating FDs of Lamb waves under undamaged and damaged conditions as $d$ and $d^{\prime}$, damage index can be defined as

$$
D I=1-\frac{d^{\prime}}{d}
$$




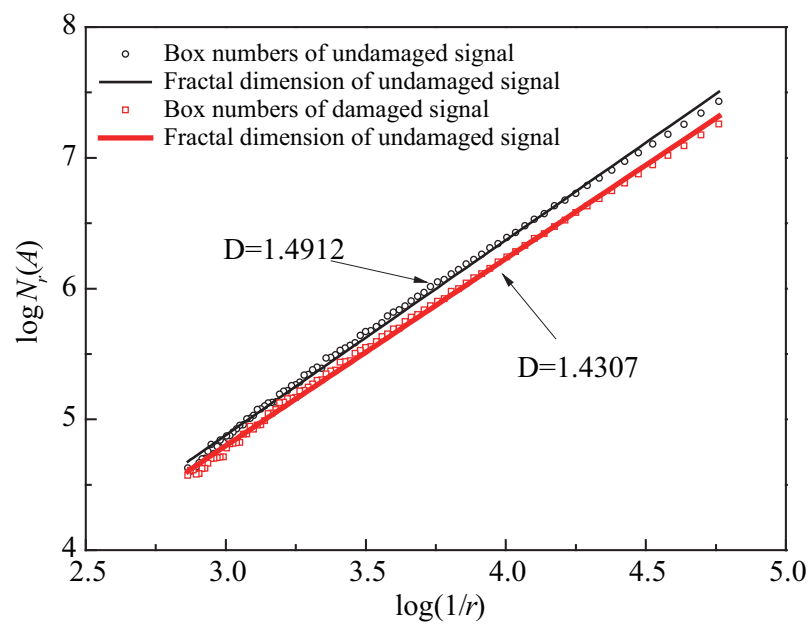

Fig. 2. Fractal dimension by least square fitting method.

As shown in Fig. 1, a typical five-peaked, Hanning windowed sinusoidal toneburst is considered as the undamaged signal, and $70 \%$ of this signal is labeled as the damaged one. FDs of both undamaged and damaged signals are calculated by comparing with each other under the same meshes. The signal domain is divided by boxes with different sizes. For each size $r_{i}$, the number of boxes covered by signal curves is noted as $N_{r i}(A)$. Figure 2 gives different values $\left(-\log r_{i}, \log N_{r i}(A)\right)$ under different box-sizes. Least squares method is used to fit data points and get the slop, i.e., FD of Lamb wave signal. FDs of undamaged and damaged signals in Fig. 1 are calculated as $d=$ 1.4912 and $d^{\prime}=1.4307$, and then damage index can be got as $D I=0.04057$.

\section{Imaging algorithm}

A reconstruction algorithm for probabilistic inspection of damage (RAPID) has been developed to study damage detection, grown monitoring and location mapping with transducer arrays $[21,22]$. This method removes some shortcomings of the past computer tomography methods, which is generally considered as time consuming and insensitive for sparse transducer array. The present paper introduces an image-enhancing factor and proposed an enhanced reconstruction algorithm for probabilistic inspection of damage (ERAPID). Compared with RAPID, ERAPID will give a more perfect image to show the damage in complex structures.

Based on damage index of each transmitter-receiver path calculated by FDs, the probabilities of each pixel point is summed as follows

$$
P(x, y)=\left(\sum_{i=1}^{k} p_{i}(x, y)\right)^{\alpha}=\left(\sum_{i=1}^{k} D I_{i} \frac{\beta-R_{i}}{\beta-1}\right)^{\alpha}
$$

where $P(x, y)$ is the damage present probability at pixel point $(x, y), k$ is the total number of paths, $p_{i}(x, y)$ is the damage present probability at pixel point $(x, y)$ calculated by the $i$ th path, and $D I_{i}$ is damage index of the $i$ th path by Eq. (5). $\beta$, a constant greater than 1, is a scaling factor to control influence region of a transmitter-receiver path. $\alpha$ is an image enhancement factor, which can increase the image contrast of damaged domain. $R_{i}$ can be written as

$$
R_{i}= \begin{cases}R D_{i}, & R D_{i}<\beta \\ \beta, & R D_{i} \geqslant \beta\end{cases}
$$

where $R D_{i}$ is the ratio of the total distance measured from the point $(x, y)$ to the transmitter $\left(x_{a i}, y_{a i}\right)$ and to the receiver $\left(x_{s i}, y_{s i}\right)$, to the distance between the transmitter and the receiver

$$
R D_{i}=\frac{\sqrt{\left(x-x_{a i}\right)^{2}+\left(y-y_{a i}\right)^{2}}+\sqrt{\left(x-x_{s i}\right)^{2}+\left(y-y_{s i}\right)^{2}}}{\sqrt{\left(x_{a i}-x_{s i}\right)^{2}+\left(y_{a i}-y_{s i}\right)^{2}}}
$$




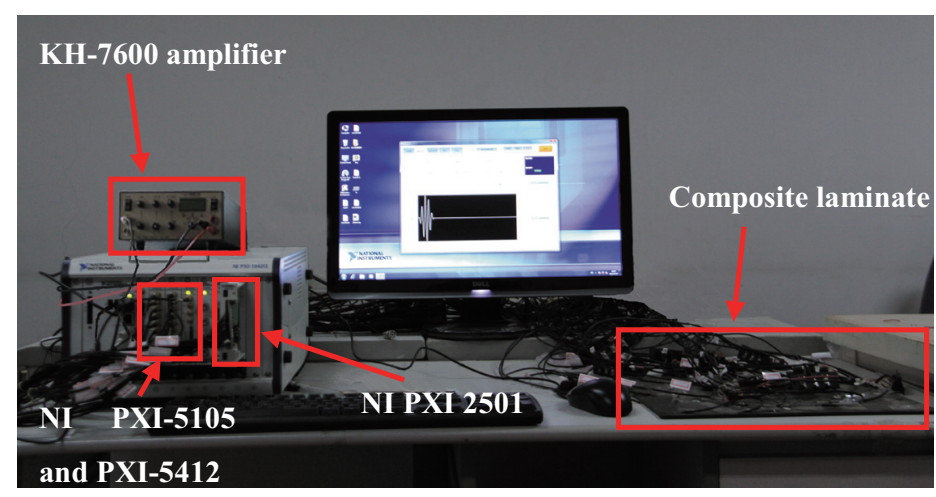

Fig. 3. A multi-channel PZT transducer-array scanning system.

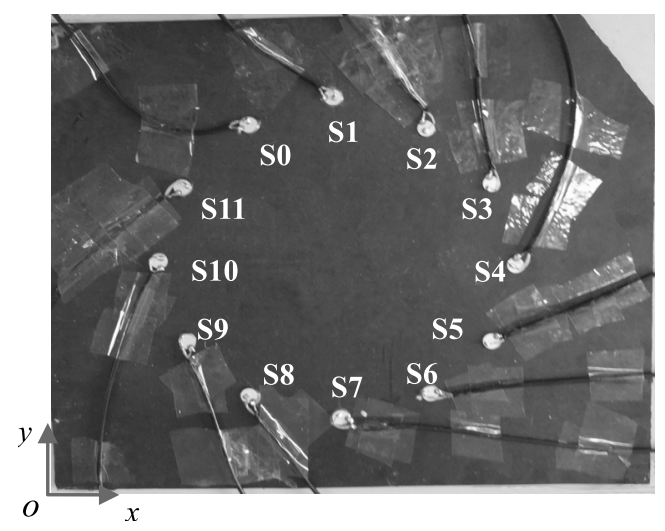

Fig. 4. A composite structure and transducer array.

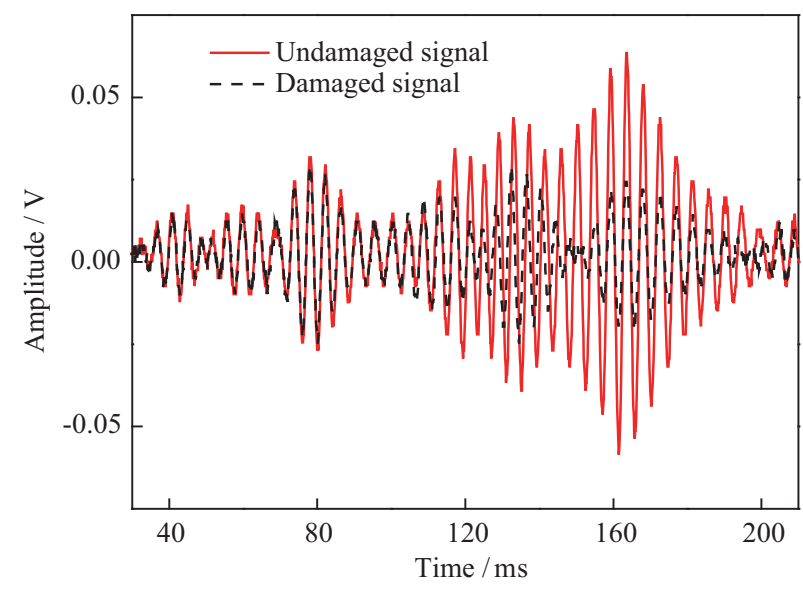

Fig. 5. Lamb wave signals in the path S2-S10.

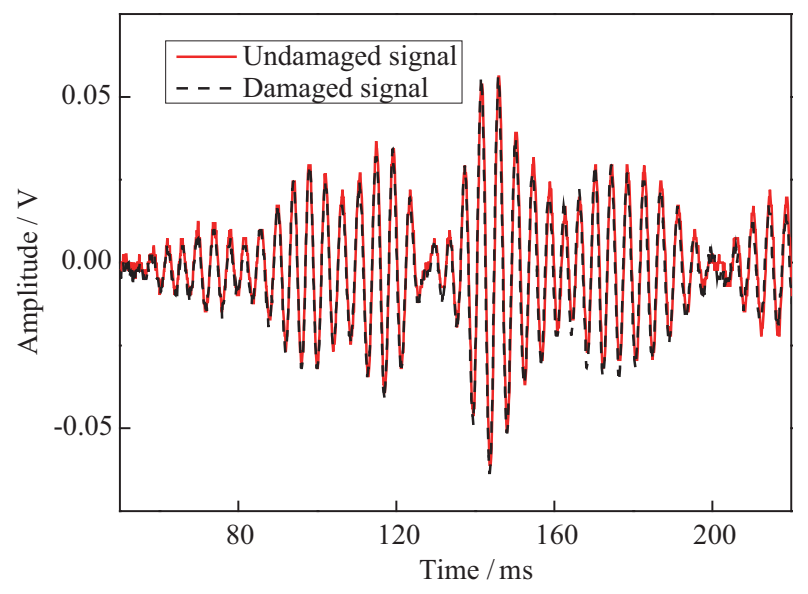

Fig. 6. Lamb wave signals in the path S2-S8.

The contours of the distribution estimation are a set of ellipses with the transmitter and receiver being at two focal points. When $R D_{i} \geqslant \beta$, i.e., the point $(x, y)$ is on or out of the ellipse, $p_{i}(x, y)=0$. when $R D_{i}=1$, i.e., the point $(x, y)$ is on the straight line between the transmitter and receiver, $p_{i}(x, y)=D I_{i}$.

\section{Experimental study}

Experimental tests are conducted to examine the effectiveness of the developed method. As shown in Fig. 3, the test set-up system, a multi-channel PZT transducer-array scanning system, consists of a KH-7600 wideband charge amplifier, two NI PXI-5105 data acquisition (DAQ) boards with 8-chanels simultaneously sampled at 12-bit resolution, a NI PXI-5412 arbitrary function generator, a NI PXI-2501 multiplexer/matrix module and NI LABVIEW software. A composite laminate and a stiffened composite panel, as two different kinds of complex structures, are used in the tests. To reduce the experiment cost, artificial damages rather than real damages are introduced with bolts affixed on the surface of structures. Just as real damages scattering Lamb wave, the geometry continuum of structures is interrupted by bolts.

In the following experiments, 12 PZT transducers denoted by S0 to S11 are mounted to form a circular transducer array to measure Lamb wave in structures. The diameter of the PZT transducer is $10 \mathrm{~mm}$ and the thickness is $1 \mathrm{~mm}$. Each PZT transducer could be acted as both transmitter and receiver, thus there are totally 132 transmitter-receiver paths, including S0 to S1-S11, S1 to S0, S2-S11, etc. A five-peaked, Hanning windowed sinusoidal toneburst, with central frequency $200 \mathrm{kHz}$, is applied into NI PXI-5412 arbitrary function generator as the exciting signal. 


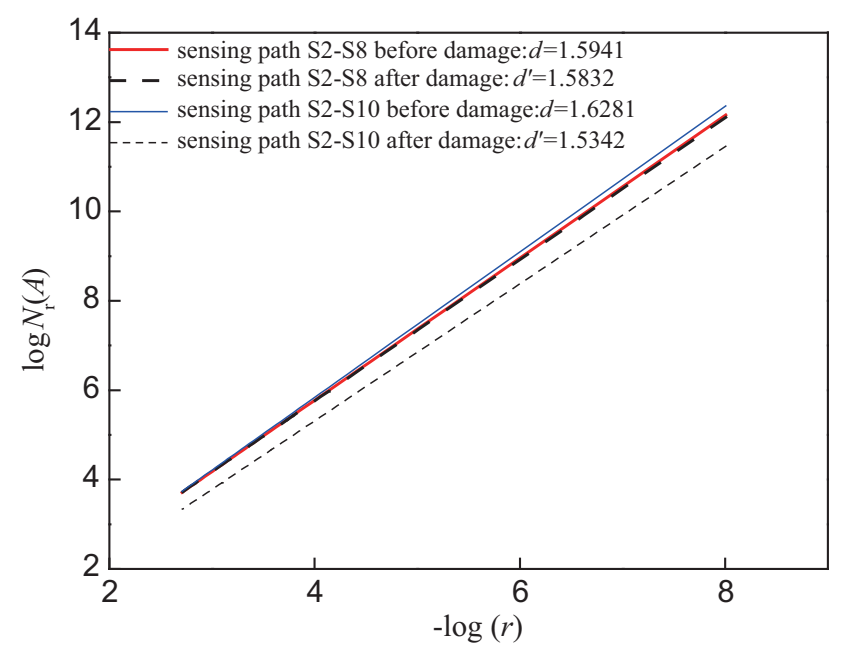

Fig. 7. Fractal dimensions of transmitter-receiver paths S2-S8 and S2-S10.

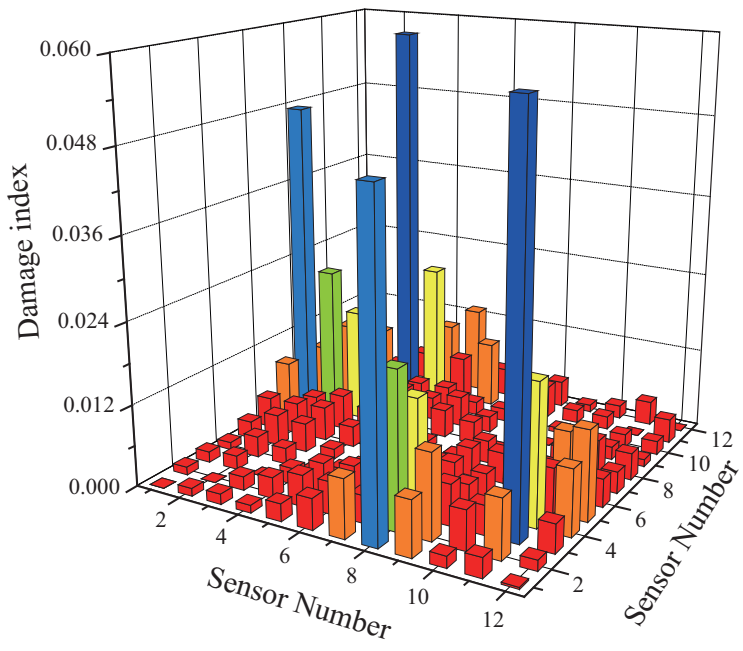

Fig. 8. Damage indices of all paths.

The signal amplified by KH-7600 drives one of PZT transducers to generate transient Lamb wave into plate-type structures. NI PXI-2501 multiplexer/matrix module is developed to be an automatic changing-channel switch, which can select each PZT transducer as transmitter spontaneously. The response wave signals are then received by other PZT transducers and saved by the NI PXI-5105 DAQ board, whose sampling rate is set as $10 \mathrm{MHz}$ and sampling point number is 5000 .

\subsection{Experiment for a composite laminate}

As shown in Fig. 4, a composite laminate has dimensions of $350 \mathrm{~mm} \times 300 \mathrm{~mm} \times 3 \mathrm{~mm}$. Taking the low-left corner as coordinate origin, the transducer array, with diameter $200 \mathrm{~mm}$ and circular center location $(170 \mathrm{~mm}$, $150 \mathrm{~mm}$ ), is mounted on one surface of the composite laminate. One bolt with radius $10 \mathrm{~mm}$ is affixed at the point $(140 \mathrm{~mm}, 185 \mathrm{~mm})$ to model the damage. The multi-channel PZT-array scanning system is employed to transmit/receive Lamb wave signals before and after damage.

Figure 5 shows Lamb wave signals transmitted by S2 and received by S10, where the path passes through the damage. It can be seen that the damaged signal is remarkably weakened compared with the undamaged signal. Conversely, it shows in Fig. 6 that there is nearly no change between undamaged and damaged signals in the spath S2-S8.

Figure 7 gives fractal dimensions of Lamb wave signals in transmitter-receiver paths S2-S10 and S2-S8 before and after damage. It can be seen from Fig. 7 that FDs of undamaged and damaged signals in the path S2-S10 greatly changed, while those of the path S2-S8 are equal to each other. The damage index of the path S2-S10 is DI= 5.767E-2, and that of S2-S8 is $D I=6.775 \mathrm{E}-3$. Figure 8 shows the histogram of damage index in each path, which denotes that if and only if there exists damage in or near some path, the damage index of this path is relatively large.

Setting parameters $\beta$ and $\alpha$ in ERAPID as 1.02 and 5, respectively, the damage image is given in Fig. 9. The circle denotes the real damage. It can be seen that the identified damage shows a good agreement with the real damage in location and size.

\subsection{Experiment for a stiffened composite panel}

As shown in Fig. 10, a stiffened composite panel with dimensions $900 \mathrm{~mm} \times 480 \mathrm{~mm} \times 3 \mathrm{~mm}$ is used in the test. The transducer array, with diameter $200 \mathrm{~mm}$, is mounted on a local domain of the stiffened panel to monitor damage state. Taking the circle center of the transducer array as coordinate origin, a bolt with radius $10 \mathrm{~mm}$ is affixed at the coordinate point $(48 \mathrm{~mm}, 29 \mathrm{~mm})$. The process of data acquisition is conducted by the multi-channel PZT transducer-array scanning system. 


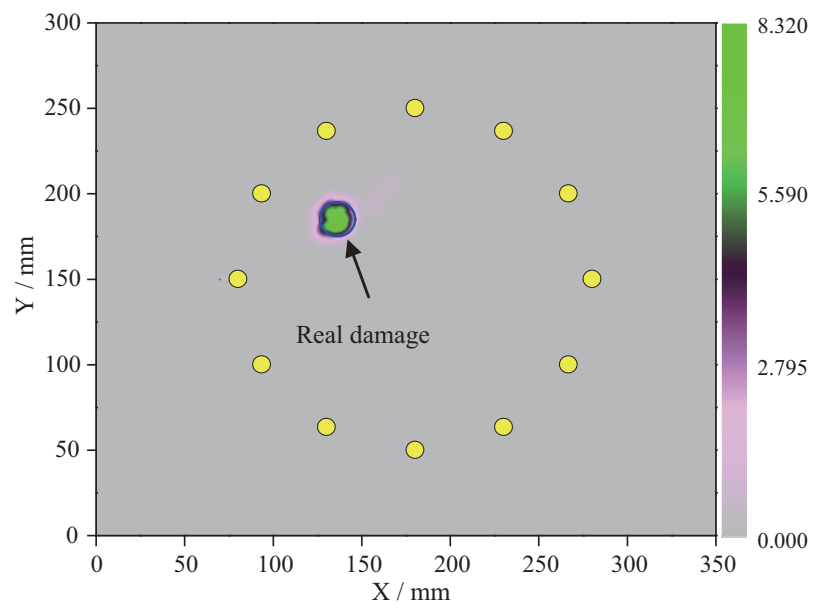

Fig. 9. Damage image of the composite laminate.

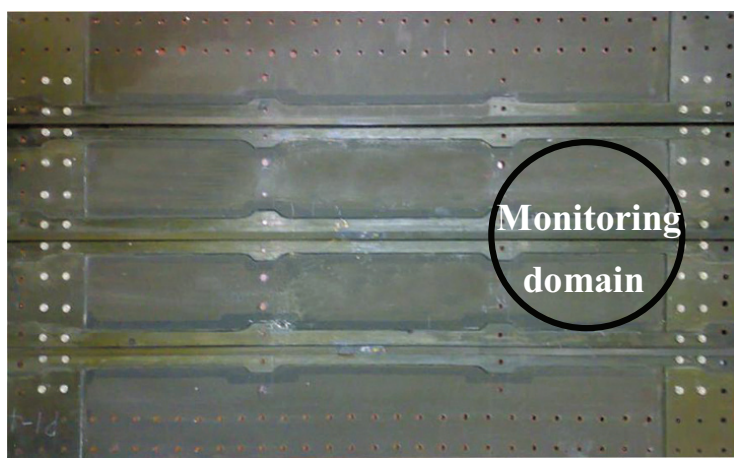

(a)

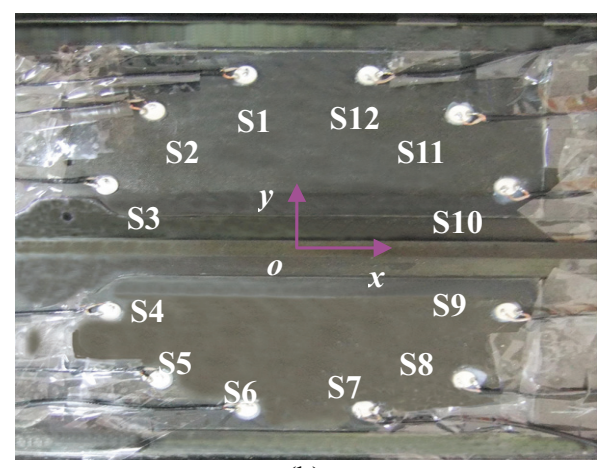

(b)

Fig. 10. Stiffened composite panel and transducer array: (a) Stiffened panel; (b) Monitoring area.

FDs of response signals under undamaged and damaged states are calculated respectively. Then damage indices are extracted and shown in Fig. 11. It can be shown that although ribs influence Lamb wave propagation, damage indices of transmitter-receiver paths which pass through the damage are also larger than those not passing through the damage. According to setting parameters $\beta$ and $\alpha$ as 1.02 and 5 in ERAPID, the damage image is given in Fig. 12. The result clearly shows the capability of the developed approach to identify the right location and size of the damage.

\subsection{Parametric study}

The composite laminate in Fig. 4 is employed to study the influence of the scaling factor $\beta$ and the image enhancement factor $\alpha$ on the imaging result and accuracy. This will contribute to the definition of these two parameters in real use.

The scaling factor $\beta$ is firstly discussed. Figure 13 shows image results when $\beta$ equals $1.001,1.02,1.04,1.06$, 1.08 and 1.1, respectively. It indicates that $\beta$ affects the size of identified damage, i.e., accuracy of image. It can be concluded that the greater the scaling factor, the larger the size of damage. When $\beta$ is equal to 1.02 , the size of identified damage is most identical to real damage.

The image enhancement factor $\alpha$ is also considered. Figure 14 shows that the image enhancement factor $\alpha$ not only affects the size of identified damage, but also forms some shapes of transmitter-receiver paths which may interrupt the quality of the damage image. It can be concluded that when $\alpha$ is equal to 5, the image has a perfect quality and the size of identified damage keeps in accordance with real damage. 


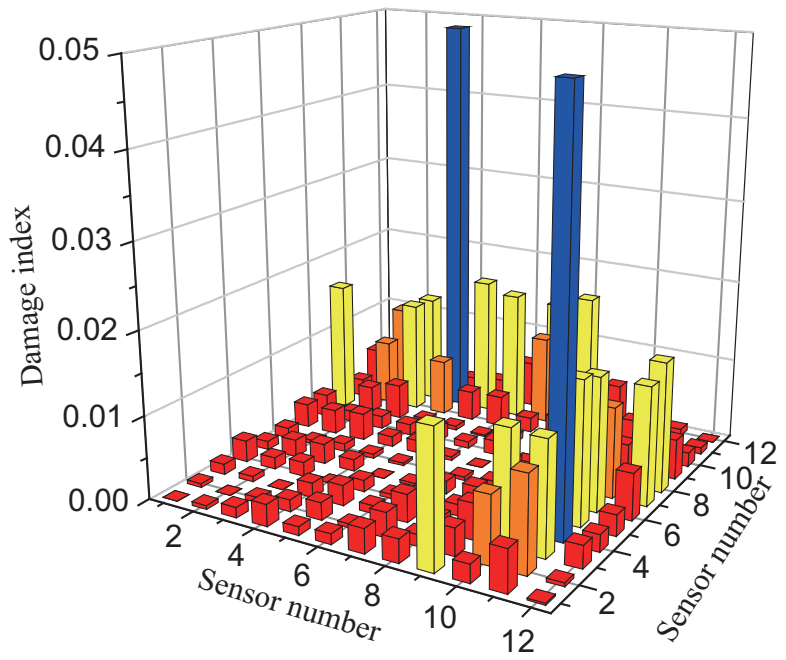

Fig. 11. Damage index in different paths of the stiffened composite panel.

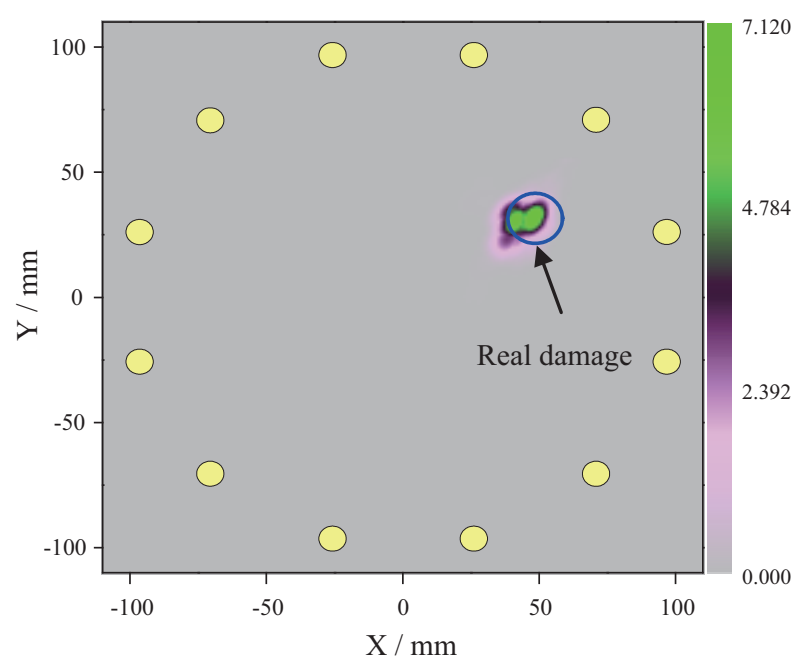

Fig. 12. Damage image for the stiffened composite panel.

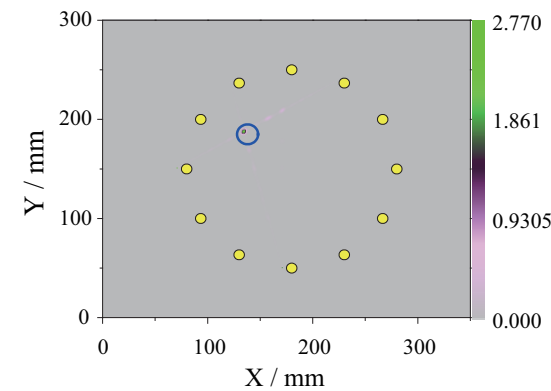

(a)

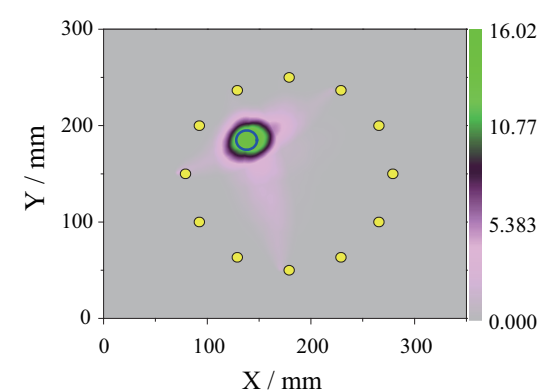

(d)

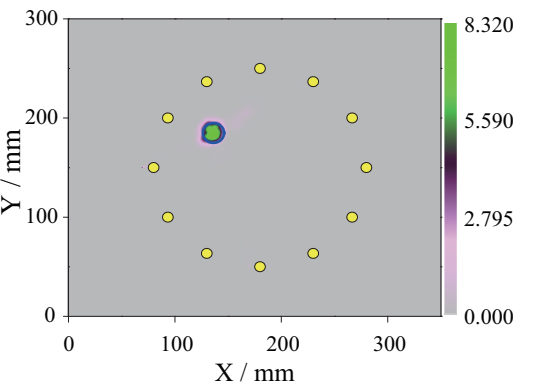

(b)

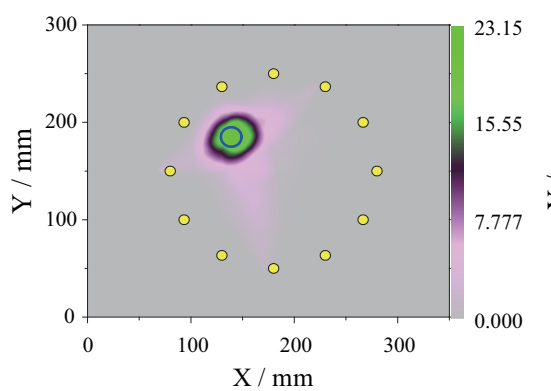

(e)

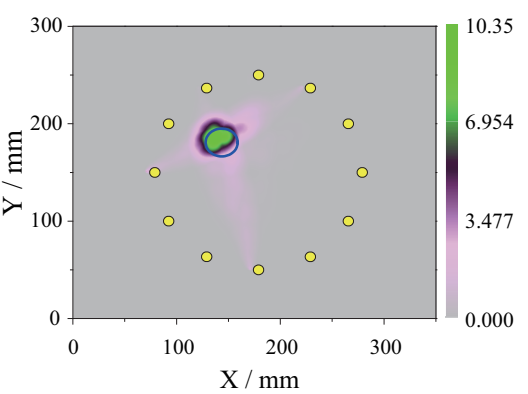

(c)

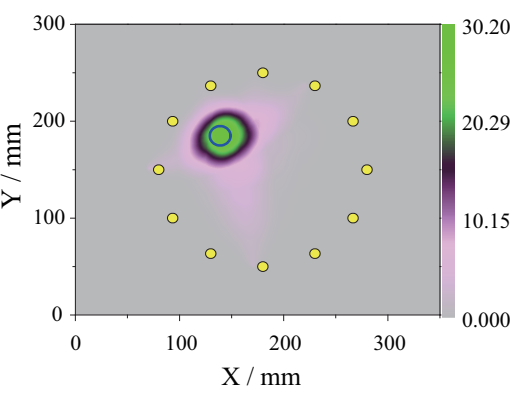

(f)

Fig. 13. The influence of the scaling factor $\beta$ on the imaging result. (a) $\beta=1.001$; (b) $\beta=1.02$; (c) $\beta=1.04$; (d) $\beta=1.06$; (e) $\beta=1.08$; (f) $\beta=1.1$.

\subsection{The capability to identify multi-damage}

Setting two damages in the composite laminate in Fig. 4, the capability of the developed algorithm to identify multi-damage is evaluated. One damage is modeled by a bolt at coordinate point $(140 \mathrm{~mm}, 185 \mathrm{~mm})$ with radius $10 \mathrm{~mm}$, and another is at (192 $\mathrm{mm}, 134 \mathrm{~mm}$ ) with radius $5 \mathrm{~mm}$.

Figure 15 shows damage indices of all transmitter-receiver paths, which show more paths passing through damages compared with one damage condition in Fig. 11. Figure 16 gives the image of two damages, from which it can 


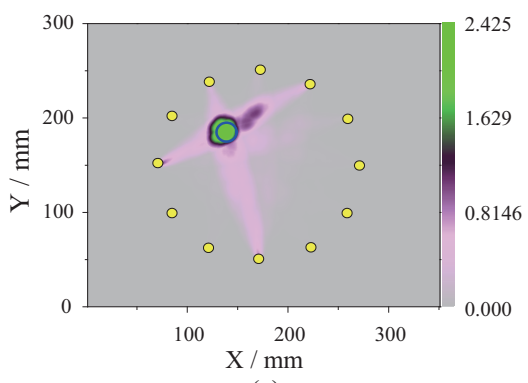

(a)

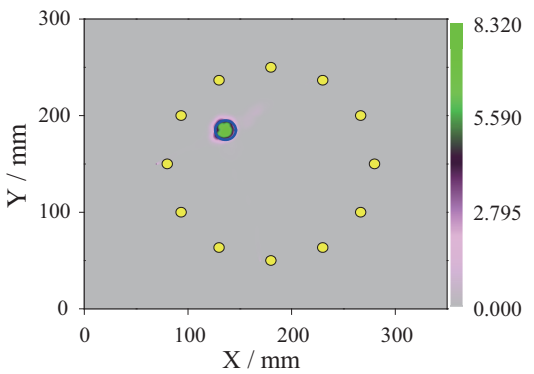

(c)

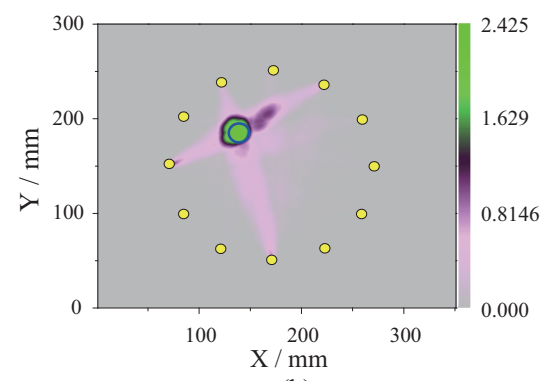

(b)

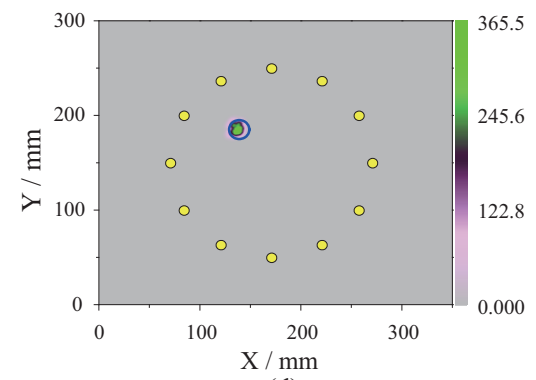

(d)

Fig. 14. The influence of the image enhancement factor $\alpha$ on the imaging result. (a) $\alpha=1$; (b) $\alpha=3$; (c) $\alpha=5$; (d) $\alpha=10$.

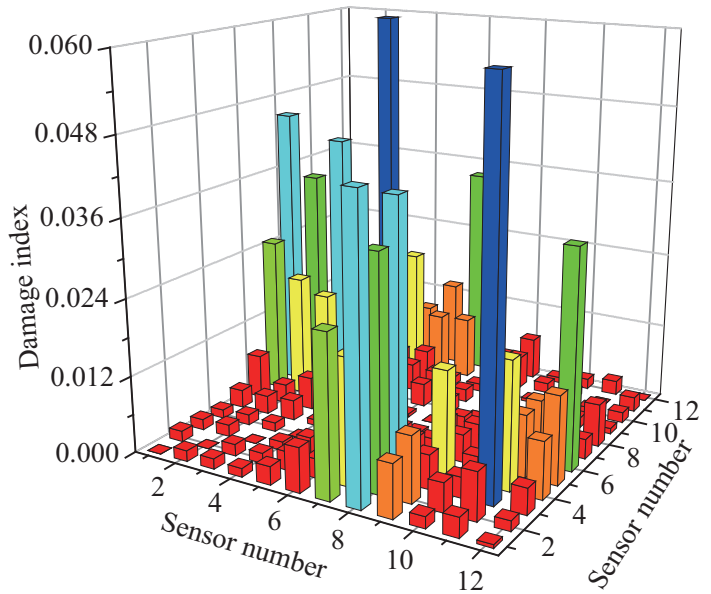

Fig. 15. Damage indices of the composite laminate with two damages.

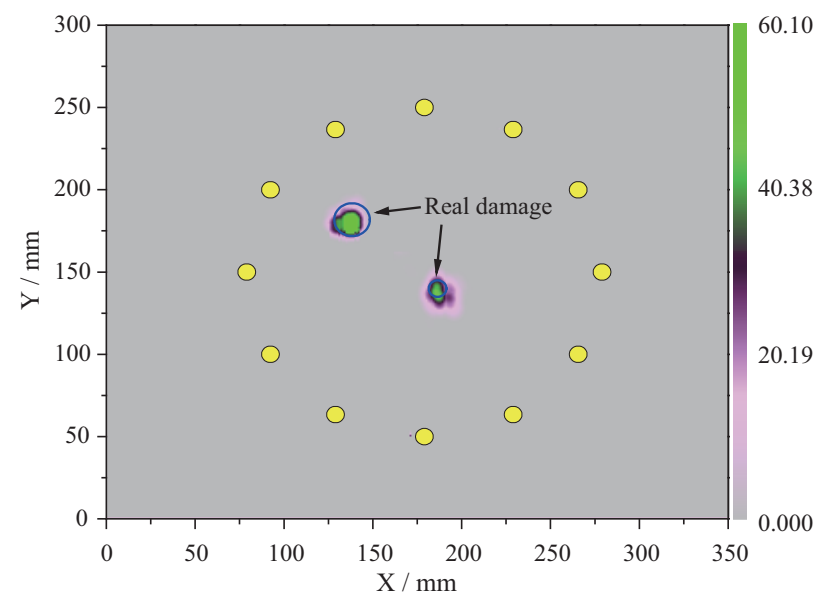

Fig. 16. Damage image of the composite with two damages.

be seen that identified damages distinctly exhibit the location and size of real damages. It denotes that the developed algorithm has the ability to identify multiple damages.

\section{Conclusion}

Combing fractal dimension and an enhanced reconstruction algorithm for probabilistic inspection of damage, a quantitative damage imaging algorithm has been developed. The developed algorithm has been verified to be able to predict the location and size of the damage in the tests of a composite laminate and a stiffened composite panel conducted via a multi-channel PZT transducer-array scanning system developed by the author's team. It has been also demonstrated that the algorithm has the capability to identify multi-damage. The influence of parameters in the 
developed algorithm on the imaging quality and accuracy is studied, which may provide some references for real use of the developed algorithm.

\section{Acknowledgments}

This research is supported by the National Natural Science Foundation of China (Grant No. 11172128 and Grand No. 61161120323), the Specialized Research Fund for the Doctoral Program of Higher Education of China (Grant No.20123218110001), the 'Six Talent Peak' Project of Jiangsu Province (Grant No. 2010-JZ-004) and a Project Funded by the Priority Academic Program Development of Jiangsu Higher Education Institutions.

\section{References}

[1] V. Giurgiutiu and C. Soutis, Enhanced composites integrity through structural health monitoring, Applied Composite Materials 19 (2012), $813-829$.

[2] C.M. Yeum, H. Sohn, J.B. Ihn et al., Instantaneous delamination detection in a composite plate using a dual piezoelectric transducer network, Composite Structures 94 (2012), 3490-3499.

[3] N. Quaegebeur, P. Micheau, P. Masson et al., Methodology for optimal configuration in structural health monitoring of composite bonded joints, Smart Materials and Structures 21 (2012), 105001.

[4] L. Wang and F.G. Yuan, Damage identification in a composite plate using prestack reverse-time migration technique, Structural Health Monitoring 4 (2005), 195-211.

[5] L. Zhou, F.G. Yuan and W. Meng, A pre-stack migration method for damage identification in composite structures, Smart Structures and Systems 3 (2007), 439-454.

[6] H. Sohn, H.W. Park, K.H. Law et al., Damage detection in composite plates by using an enhanced time reversal method, Journal of Aerospace Engineering, ASCE 20(3) (2007), 141-151.

[7] H. Sohn, H.W. Park, K.H. Law et al., Combination of a time reversal process and a consecutive outlier analysis for baseline-free damage diagnosis, Journal of Intelligent Material Systems and Structures 18(4) (2007), 335-346.

[8] L. Qiu, S.F. Yuan, X.Y. Zhang et al., A time reversal focusing based impact imaging method and its evaluation on complex composite structures, Smart Materials and Structures 20(10) (2011), 105014.

[9] X. Miao, D. Wang, L. Ye et al., Identification of dual notches based on time-reversal lamb waves and a damage diagnostic imaging algorithm, Journal of Intelligent Material Systems and Structures 22 (2011), 1983-1992.

[10] P. Malinowski, T. Wandowski, I. Trendafilova et al., A phased array-based method for damage detection and localization in thin plates, Structural Health Monitoring 8 (2008), 5-15.

[11] V.T. Rathod, N. Chakraborty and D.R. Mahapatra, Linear phased array of piezoelectric transducers for delamination monitoring in a composite laminate using Lamb waves, Proc SPIE 7982, Smart Sensor Phenomena, Technology, Networks, and Systems (2011), 798217.

[12] X. Zhao, R.L. Royer, S.E. Owens et al., Ultrasonic lamb wave tomography in structural health monitoring, Smart Materials and Structures 20(10) (2011), 105002.

[13] D. Wang, L. Ye and Y. Lu, A probabilistic diagnostic algorithm for identification of multiple notches using digital damage fingerprints (DDFs), Journal of Intelligent Material Systems and Structures 20 (2009), 1439-1450.

[14] R. Lopes and N. Betrouni, Fractal and multifractal analysis: A review, Medical Image Analysis 13 (2009), 634-649.

[15] B.S. Raghavendra and D.D. Narayana, Computing fractal dimension of signals using multiresolution boxcounting method, International Journal of Information and Mathematical Sciences 6 (2010), 50-65.

[16] H. Li, Y. Huang, J. Ou et al., Fractal dimension-based damage detection method for beams with a uniform cross-section, Computer-Aided Civil and Infrastructure Engineering 26 (2011), 190-206.

[17] R. Bai, M. Cao, Z. Su et al., Fractal dimension analysis of higher-order mode shapes for damage identification of beam structures, Mathematical Problems in Engineering Volume 2012 (2012), 454568.

[18] L.J. Hadjileontiadis, A.E. Doukab and A. Trochidisc, Fractal dimension analysis for crack identification in beam structures, Mechanical Systems and Signal Processing 19 (2005), 659-674.

[19] B. Mandelbrot, Fractals: Form, chance and dimension, Freeman, New York (1977).

[20] B. Mandelbrot, The fractal geometry of nature, Freeman, New York (1983).

[21] T.R. Hay, R.L. Royer, H.D. Gao et al., A comparison of embedded sensor lamb wave ultrasonic tomography approaches for material loss detection, Smart Materials and Structures 15 (2006), 946-951.

[22] X. Zhao, H. Gao, G. Zhang et al., Active health monitoring of an aircraft wing with embedded piezoelectric sensor/actuator network: I. Defect detection, localization and growth monitoring, Smart Materials and Structures 16 (2007), 1208-1217. 

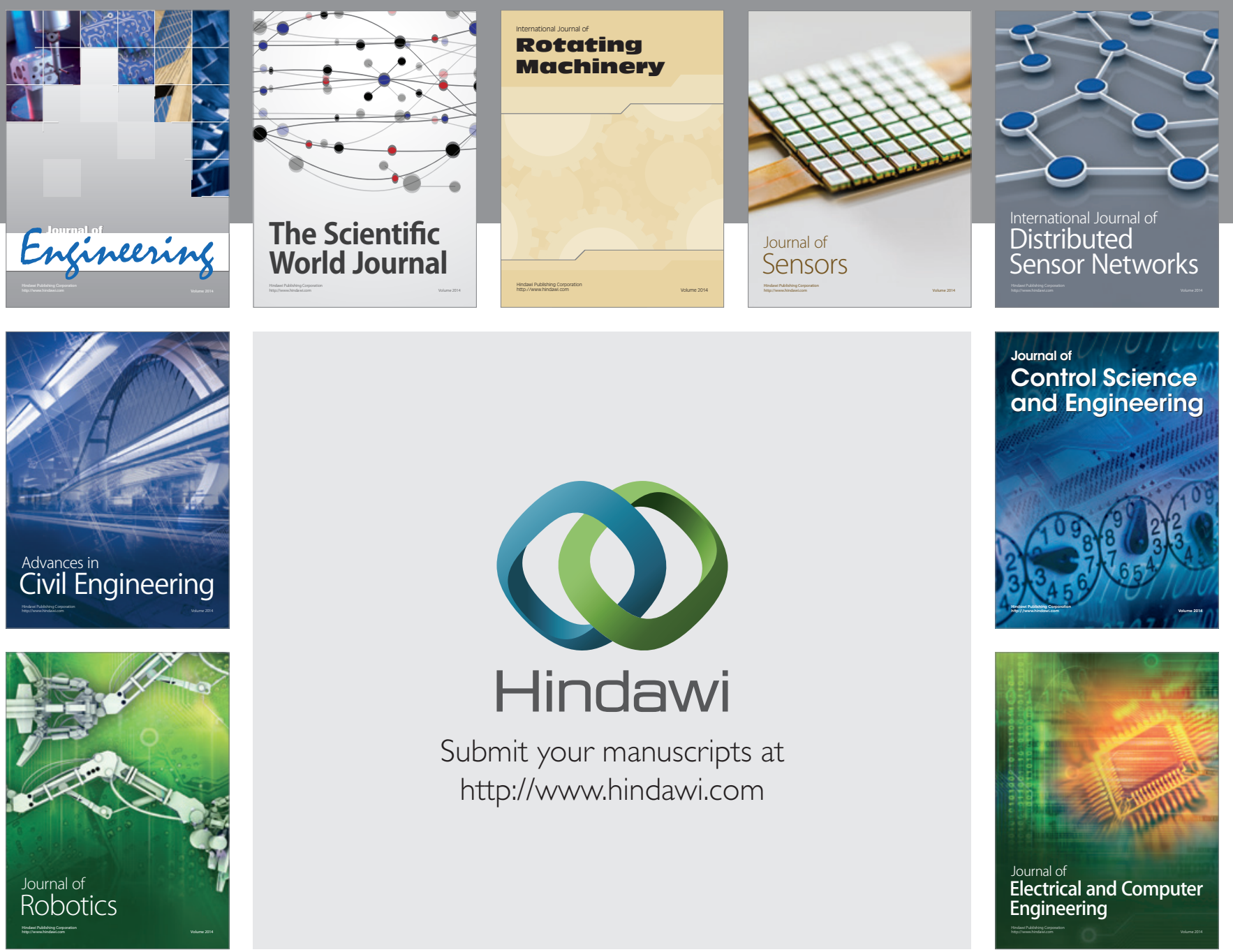

Submit your manuscripts at

http://www.hindawi.com
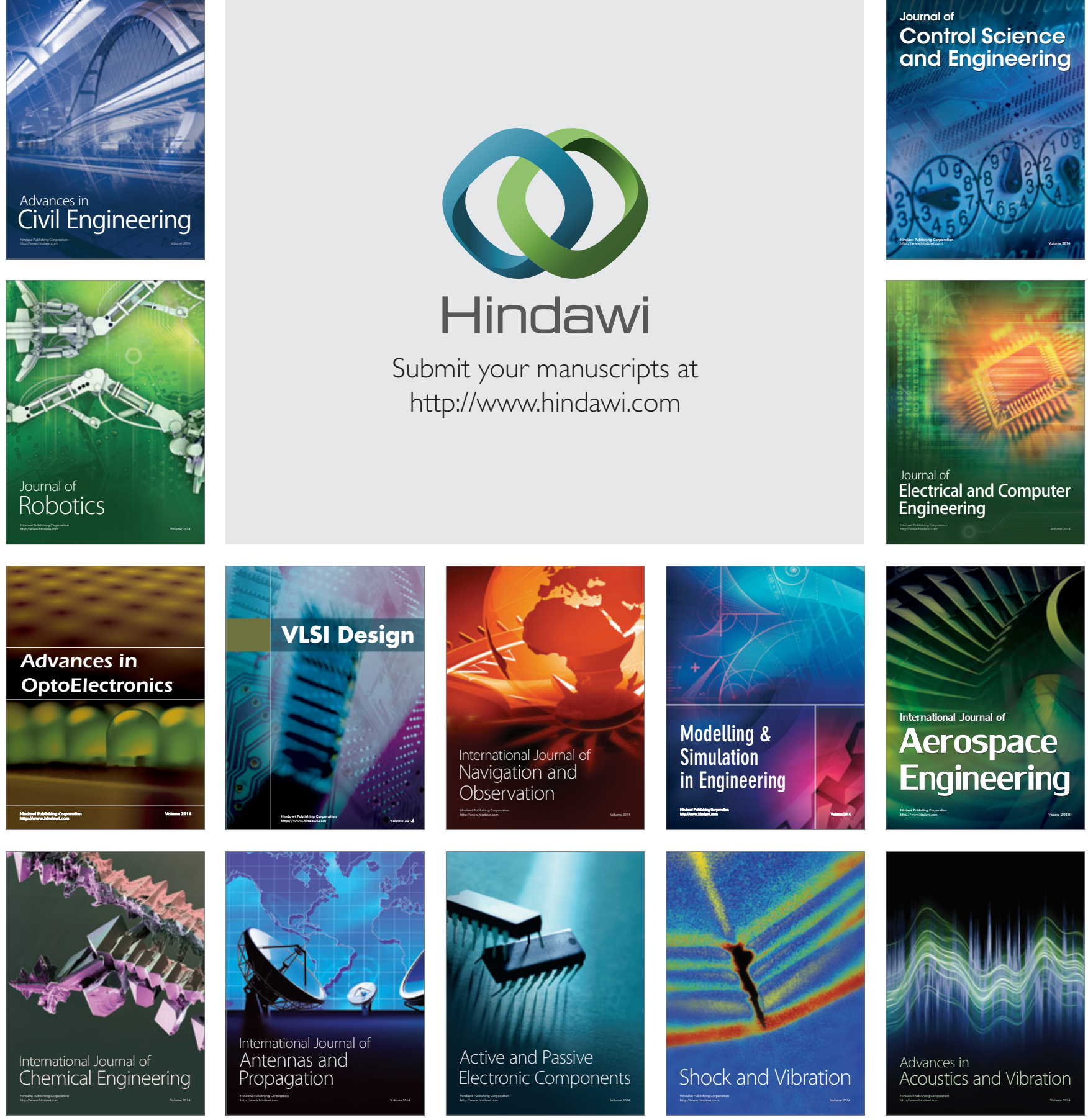